\title{
Caspase-dependent non-apoptotic processes in development
}

\author{
Yu-ichiro Nakajima ${ }^{\star, 1,2}$ and Erina Kuranaga ${ }^{\star, 2,3}$
}

Caspases are at the core of executing apoptosis by orchestrating cellular destruction with proteolytic cascades. Caspasemediated proteolysis also controls diverse nonlethal cellular activities such as proliferation, differentiation, cell fate decision, and cytoskeletal reorganization. During the last decade or so, genetic studies of Drosophila have contributed to our understanding of the in vivo mechanism of the non-apoptotic cellular responses in developmental contexts. Furthermore, recent studies using C. elegans suggest that apoptotic signaling may play unexpected roles, which influence ageing and normal development at the organism level. In this review, we describe how the caspase activity is elaborately controlled during vital cellular processes at the level of subcellular localization, the duration and timing to avoid full apoptotic consequences, and also discuss the novel roles of non-apoptotic caspase signaling in adult homeostasis and physiology.

Cell Death and Differentiation (2017) 24, 1422-1430; doi:10.1038/cdd.2017.36; published online 19 May 2017

Facts

- Caspase activation is locally regulated in subcellular compartments so that cellular remodeling during terminal differentiation is accomplished without causing cell death.

- Transient caspase activity is utilized for cell fate determination in mammalian stem cells and Drosophila neural progenitor cells in which no organelle degeneration occurs.

- Temporal control of the caspase activity mediated by IAP turnover regulates the cytoskeletal dynamics during cellular morphogenesis and cell migration in Drosophila.

- Caspase-mediated signaling at the organism level affects animal-wide phenotypes, such as ageing and normal development in C. elegans.

\section{Open Questions}

- What triggers caspase activation in nonlethal cellular processes in diverse developmental contexts?

- How is caspase activation spatiotemporally controlled and how are specific substrates recognized by active caspases during non-apoptotic processes?

- How does caspase signaling integrate cellular responses and affect organism-level phenotypes?

- What does the past-caspase activation in normal healthy cells tell us? Are there novel non-apoptotic functions of caspases or does developmental anastasis occur as a physiological event?
Caspases, a family of cysteine proteases, are highly conserved throughout the metazoa and are mostly known as executioners of apoptosis. Apoptosis is a genetically encoded suicide program that manifests distinct morphological features including cell shrinkage and nuclear fragmentation followed by phagocytic clearance. ${ }^{1}$ During development, apoptosis is widely observed and it plays a pivotal role in sculpting or removing tissues by eliminating unwanted cells. ${ }^{2-4}$ Apoptosis also assists morphogenesis and organogenesis by providing mechanical forces. ${ }^{5-7}$ In contrast, the inhibition of apoptosis causes developmental defects, such as aberrant heart formation and exencephaly. ${ }^{8}$ Furthermore, dysregulation of cell death signaling leads to human diseases including cancer and inflammatory disorders. ${ }^{9-11}$ Thus, caspase-mediated apoptosis is a fundamental cellular response in development, homeostasis, and pathophysiology.

Although caspases have been recognized as killer enzymes, studies over the last 15 years have indicated that the same enzymes also have vital functions. Such non-apoptotic functions of caspases are diverse, ranging from immune response, cell proliferation, cellular remodeling, cell fate determination to cytoskeletal reorganization. ${ }^{12-20}$ Studies using genetically tractable animals, especially the fruit fly Drosophila melanogaster, have provided mechanistic insights into non-apoptotic caspase signaling, which is under local, transient or temporal control in different developmental scenarios. In addition, recent studies using the nematode $C$. elegans have revealed novel functions of caspase signaling at the organism level. In this review, we exclusively focus on caspase-dependent nonapoptotic processes in development, aiming to introduce the

${ }^{1}$ Frontier Research Institute for Interdisciplinary Sciences (FRIS), Tohoku University, Sendai 980-8578, Japan; ${ }^{2}$ Laboratory for Histogenetic Dynamics, Graduate School of Life Sciences, Tohoku University, Sendai 980-8578, Japan and ${ }^{3}$ Laboratory for Histogenetic Dynamics, RIKEN Center for Developmental Biology, Kobe 650-0047, Japan ${ }^{*}$ Corresponding author: Y-I Nakajima, Frontier Research Institute for Interdisciplinary Sciences, Tohoku University, Sendai 980-8578, Japan. Tel: +81 22 795 6701; Fax: +81 22795 6701; E-mail: yuichiro.nakajima.d2@tohoku.ac.jp

or E Kuranaga, Laboratory for Histogenetic Dynamics, Graduate School of Life Sciences, Tohoku University, Sendai 980-8578, Japan. Tel: +81 22 795 6709; Fax: +81 22 795 6709; E-mail: erina.kuranaga.d1 @ tohoku.ac.jp

Received 06.1.17; revised 17.2.17; accepted 20.2.17; Edited by E Arama; published online 19.5.17 
progress in understanding the mechanism that controls the nonlethal function of caspases and to discuss future directions of this field of research. As has been previously proposed, ${ }^{21}$ we herein consider development as the entire life span of the organism, including the so-called 'egg to organism' and the following adulthood stage.

\section{Conserved Caspase-Mediated Pathway in Development}

The core caspase signaling pathway has been studied using model animals, $C$. elegans, Drosophila, and the mouse. The first cell death gene was identified by the genetic study of C. elegans, in which ced-3 mutant exhibited the survival of 131 cells that are destined to die. ${ }^{22}$ In $C$. elegans, four genes (ced-3, ced-4, ced-9, and egl-1) control apoptotic cell death as a linear pathway; CED-3 is a caspase and CED-4 is its activator, whereas CED-9 and EGL-1 belong to the BCL-2-like anti-apoptotic proteins and BH3-only pro-apoptotic proteins, respectively. When cells undergo apoptosis, EGL-1, which is transcriptionally upregulated, displaces CED-9 from its complex with CED-4 at mitochondria, and the released CED-4 interacts with CED-3 to activate the proteolytic activity, resulting in cell death (Figure 1a). ${ }^{23}$

Caspases are synthesized as zymogens that are processed at selective aspartate residues for their activation. ${ }^{24}$ In flies and mammals, apoptotic caspases are subdivided into two categories, initiator caspases (ex. Dronc in fly; caspase- 9 in mammals) and effector caspases (ex. Drice and Dcp-1 in fly; caspase-3 and -7 in mammals). Initiator caspases have long amino-terminal prodomains that provide the molecular platform for caspase activation. In mammals, upon the release of cytochrome $c(\mathrm{Cyt}-c)$ from mitochondria, the adapter protein apoptosis activating factor 1 (Apaf-1) forms the apoptosome complex with Cyt- $c$ and pro-caspase-9, which initiates proteolytic cascades by cleaving the zymogens of caspases. After processing, activated caspase-3 cleaves hundreds of substrates and executes apoptotic cell death. ${ }^{25}$ Similarly, in Drosophila, Apaf-1-related killer (Ark) forms the apoptosome with the caspase-9 ortholog Dronc to activate caspase-3-like effector caspases, Drice and Dcp-1 (Figures $1 \mathrm{~b}$ and c). ${ }^{26}$

Caspase signaling is also negatively controlled by the inhibitor of apoptosis proteins (IAPs). ${ }^{27}$ Several IAPs carry E3ubiquitin ligase activity that promotes the degradation of cell death regulators by the ubiquitin-proteasome system. Upon apoptosis signaling stimuli, IAPs are inhibited by IAP antagonists, which leads to caspase activation. In Drosophila, Reaper, Hid, and Grim, called RHG proteins, which localize to mitochondria to inhibit Drosophila IAP1 (DIAP1), promote DIAP1 self-degradation, leading to the release of caspases. ${ }^{28}$ The mammalian ortholog of DIAP1 is X-linked IAP (XIAP), which has E3 ligase activity, and IAP antagonists such as Smac, HtrA2, and ARTS trigger caspase activation by binding to IAPs or promoting their degradation (Figures $1 \mathrm{~b}$ and c). 29,30

In summary, caspase activity is under tight control by both the activating and the inhibiting mechanisms, and these pathways are further regulated by different layers of upstream signaling networks. ${ }^{31}$ As illustrated in the following, nonapoptotic events appear to utilize the same set of core caspase signaling, although caspase activation is regulated
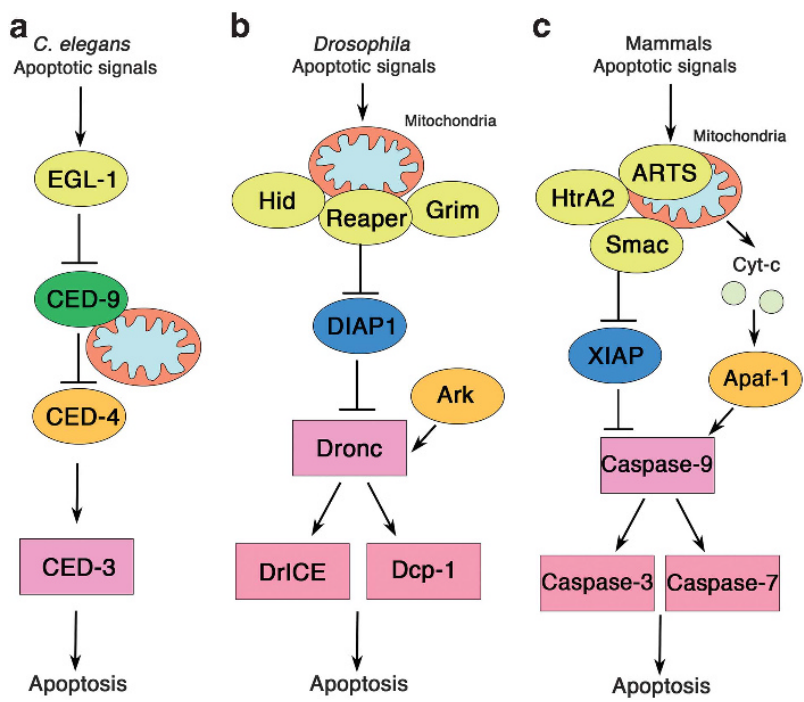

Figure 1 Conserved core caspase-signaling cascades. (a) In C. elegans, apoptotic signals transcriptionally upregulate EGL-1(BH3-only protein), of which binding to CED-9 (BCL2-family protein) releases CED-4 and consequently promotes the activation of caspase CED-3. (b) In Drosophila, different developmental signals become apoptotic stimuli and activate IAP antagonists Reaper, Hid, and Grim (RHG). $\mathrm{RHG}$ proteins localize to mitochondria and promote degradation of Drosophila inhibitor of apoptosis protein 1 (DIAP1). DIAP1 inhibits Dronc and effector caspases Drice and Dcp-1 so that the degradation of DIAP1 release caspases. Dronc and its activator Ark form the apoptosome complex, which activates downstream effector caspases. (c) In mammals, the interplay between pro-apoptotic and anti-apoptotic BCL-2 family proteins controls the release of mitochondria-localized proteins including Cyt-c and IAP antagonists such as Smac, HtrA2, and ARTS. Binding of Cyt- $C$ to the Apaf-1 promotes apoptosome assembly, which recruits pro-caspase- 9 and facilitates the proteolytic cascade. IAP antagonists release caspase- 9 from the inhibition by XIAP, resulting in the activation of the apoptosome and the downstream effector caspases (caspase-3 and caspase-7). Functional homologous proteins across species are similarly represented by the color and shape

through its localization, duration, and timing to avoid deleterious consequences. Caspases thus have acquired the elaborate mechanisms to activate their irreversible proteolytic ability for multiple purposes.

\section{Localized Caspase Activation in Cellular Remodeling}

During apoptosis, caspases orchestrate the cellular destruction procedure by cleaving their respective substrates, allowing the demolition of cytoarchitecture as well as the degradation of cytoplasmic organelles. Such caspasemediated proteolysis can be effective for removing specific organelles if the caspase activity is spatially controlled inside the cells. Terminal differentiation of some vertebrate cell types, such as lens epithelial cells, keratinocytes, and erythrocytes, accompanies cellular remodeling that includes the elimination of nuclei and other organelles, in which the caspase activity is required for organelle loss. ${ }^{4,13}$ During lens fiber differentiation, caspase-3-like activity, which is detected in the equatorial epithelium, is necessary for the initiation of the differentiation and enucleation process. ${ }^{32,33}$ Therefore, a loss of organelles may be attributed to partial cellular destruction mediated by local caspase activation. Similar cellular-remodeling events occur in sperm differentiation in both mammals and flies. 
Especially, spermatid terminal differentiation in male Drosophila gives compelling evidence of a nonlethal level of localized caspase activation with cellular and molecular details.

Sperm individualization in Drosophila. Drosophila spermatogenesis occurs within individual units, called cysts, where 64 haploid spermatids are initially interconnected by cytoplasmic bridges. ${ }^{34}$ Later in sperm development, an actinbased cytoskeletal-membrane complex, 'individualization complex (IC)' is formed, which caudally slides along the spermatid from the head to the tail, disconnecting the cytoplasmic bridges and separating the syncytium into individual sperms (Figure $2 a) .{ }^{35}$ This final step of spermatogenesis, termed individualization, includes disassembling of organelles and damping most cytoplasmic components into cystic bulges, which eventually accumulates in waste bags (Figure 2a). ${ }^{34}$ Because morphological changes of spermatids accompany organelle destruction and cytoplasmic expelling, this series of events is reminiscent of apoptosis.

The pioneering work by Arama et al. ${ }^{36}$ showed that effector caspase activity is required for proper sperm individualization processes. They first identified apoptotic-like corpses in cystic bulges and waste bags during spermatid individualization, whereas caspase-3-like activity is detected once IC is formed and it is highest in cystic bulges, but undetectable in the postindividualized region. Furthermore, both in cultured testes and in vivo spermatids, the inhibition of caspases blocked IC movement and the removal of the bulk cytoplasm. Consistent with the involvement of effector caspases, their activator, apoptosome components (Dronc, Ark and Cyt- $c$ ) are expressed in and required for individualizing spermatids. ${ }^{36-39}$ A recent report further demonstrated that Tango7, a previously identified cell death effector, ${ }^{40}$ physically interacts with Dronc and Ark, and regulates the apoptosome-dependent caspase activity during sperm differentiation. ${ }^{41}$ Of note, spermatids individualization defects are often correlated with male sterility, implying that caspase activity during sperm differentiation is critical for fertility. Collectively, these studies suggest that multiple components of the caspase signaling pathway tightly regulate non-apoptotic caspase activation in individualizing spermatids.

How is the caspase activity in spermatids spatially controlled and maintained at a certain level to avoid cell death while allowing cellular remodeling? In addition to pro-apoptotic genes, the giant IAP-like protein dBruce was identified to protect spermatids from excessive caspase activity that causes nuclear degeneration. ${ }^{36}$ From genetic screening, a testis-specific isoform of Cullin-3 was identified to be a component of an E3-ubiquitin ligase complex. ${ }^{42}$ Cullin-3 interacts with the small RING protein Roc1b and BTBcontaining protein KIhl10, forming a Cullin-3-based RING ubiquitin ligase (CRL3) that is required for effector caspase activation. Subsequent work from the same group identified an inhibitor of the CRL3 complex, Soti, which can compete with dBruce to bind KIhl10 protein. ${ }^{43}$ Interestingly, Soti is expressed in a gradient form in the early elongating spermatids descending from the tail to the head, which is followed by dBruce protein distribution at the time of individualization (Figure 2b). The CRL3 complex and Soti
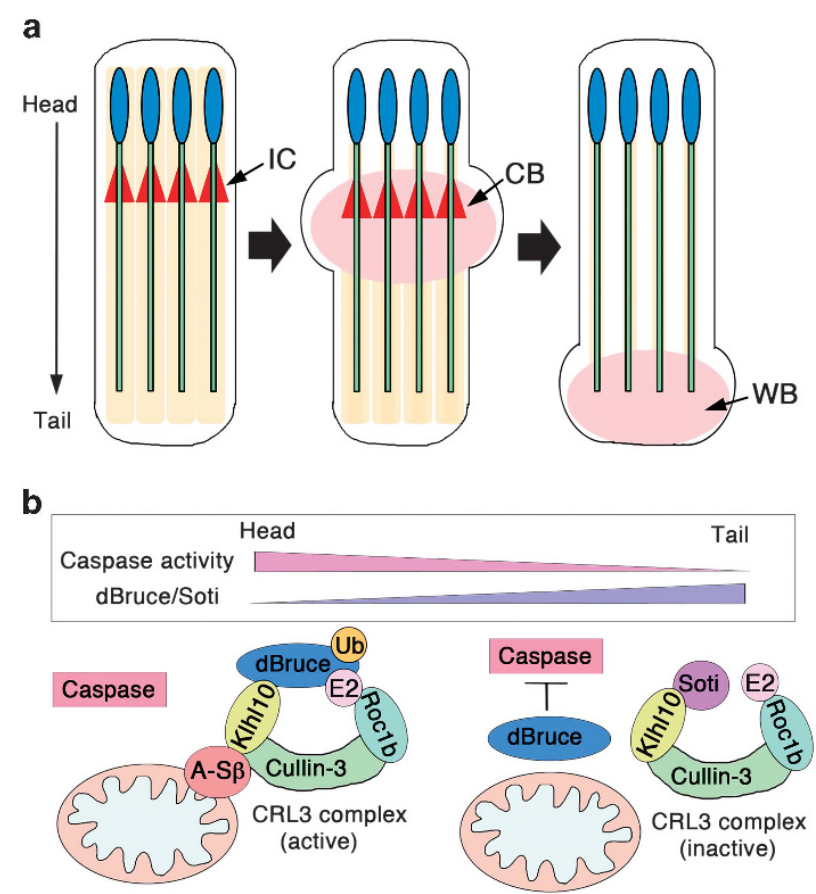

Figure 2 Localized caspase activation during Drosophila sperm individualization. (a) A schematic illustration of sperm individualization in male Drosophila. Four spermatids in a cyst are represented. Upon individualization, the individualization complex (IC, red triangle) is formed, ICs then move along the spermatid from the head to the tail direction. Along with IC movement, excess cytoplasm and organelles are removed, which accumulate in the cystic bulge (CB) and then are eventually deposited into the waste bag (WB). (b) A model of localized caspase activation in maturing spermatids. The active or inactive state of CRL 3 complex is controlled by the testis-specific isoform of A-S $\beta$ and the pseudosubstrate inhibitor Soti. The active CRL3 complex activates caspases through the ubiquitination and degradation of IAP-like protein dBruce that inhibit caspases. Soti expression forms a gradient in spermatids from the tail to the head direction, allowing the spatial gradient of dBruce distribution by inhibiting the CRL3 complex activity. The graded localization of dBruce leads to an inverse gradient of the caspase activity in spermatids from the head to the tail direction. The testis-specific A-S $\beta$ localizes to mitochondria and competes with Soti, contributing to the CRL3 activation at the surface of the mitochondria. The CRL3 complex consists of Cullin-3, KIhl10 and Roc1b that recruits an ubiquitin (Ub)-conjugating enzyme (E2)

are necessary for the spatial gradient of dBruce distribution, which in turn leads to an inverse gradient of caspase activity in spermatids from proximal to distal, allowing for the gradual accomplishment of individualization and protecting the distal region from excessive caspase activity (Figure $2 b$ ). ${ }^{43}$ The most recent study further identified a Krebs cycle component, $\beta$ subunit of the ATP-specific form of the succinyl-CoA synthetase $(A-S \beta)$ that specifically binds to the CRL3 complex. ${ }^{44}$ The long, testis-specific isoform of A-S $\beta$ is localized to mitochondria during spermatogenesis, and its expression dramatically increases in individualizing spermatids. Although A-S $\beta$ activates the CRL3 complex and is required for caspase activation, it recruits $\sim 40 \%$ of the CRL3 complex at the mitochondrial surface where their interaction occurs. This finding suggests that the mitochondria-localized activation of the CRL3 complex reduces the rate of caspase activity by $60 \%$. Mechanistically, A-S $\beta$ antagonizes the binding of Soti to the CRL3 complex, and its role is independent of the Krebs cycle, implying a novel moonlighting function of $A-S \beta{ }^{44}$ 
Altogether, spatiotemporal restriction of the CRL3 complex ensures low levels of caspase activation that avoids the rapid activation of caspases, but allows the proper removal of the cytoplasmic contents (Figure 2b).

In murine spermatids, apoptosis-like events in subcellular compartments and caspase activity are also observed during late stage of spermatogenesis. ${ }^{45,46}$ Intriguingly, sperms from mutant mouse for Sept4 gene that encodes the IAP antagonist ARTS, exhibit the retention of cytoplasm, which is similar to the defect in Drosophila sperm individualization. ${ }^{45}$ Moreover, the mammalian Klhl10, which is required for mouse spermatogenesis, interacts with Cullin-3 that is expressed in spermatids. ${ }^{47}$ Given that the orthologous proteins function as the CRL3 complex in flies, conserved mechanisms likely regulate localized caspase activation during cellular remodeling of differentiating sperms in mammals.

\section{Transient Caspase Activation in Cell Fate Determination}

Caspase-mediated proteolysis is an irreversible process that causes apoptosis as well as cellular remodeling. The same irreversible signals can be useful for determining cell fate without causing organelle degeneration if caspases cleave only specific substrates. Caspases potentially have hundreds of substrates so that the level and the duration of caspase activity must be controlled to allow for the cleavage of a few substrates. In some mammalian stem cells, the caspase-3 activity mediates the decision of self-renewal or differentiation. ${ }^{48-51}$ In embryonic stem cells, an increased caspase activity plays a critical role in differentiation by cleaving Nanog, a core transcription factor that controls their pluripotency. ${ }^{50}$ Transient caspase activity is also required for the efficient induction of induced pluripotent stem cells from human fibroblasts, which is a process of cell fate reprogramming. ${ }^{52}$ In Drosophila neural cell lineage, the caspase activity contributes to cell fate decision, which provides a foundation of understanding the mechanism of how to control transient caspase activity and its substrate specificity.

SOP cell specification in Drosophila. Drosophila macrochaetes, or large bristles, are external sensory organs located on the adult fly notum and are derived from asymmetric cell division of sensory organ precursor (SOP) cells. SOP cells of the macrochaetes originally appear in the wing imaginal disc during larval stage and are selected from proneural clusters defined by the expression of proneural genes achaete and scute, which encode basic Helix-LoopHelix transcription factors. ${ }^{53}$ The proneural activity is crucial for SOP cell specification so that the gene regulatory network tightly controls the level and timing of proneural gene expression. ${ }^{54}$ One of the essential regulators is the Wingless $(\mathrm{Wg}) / \mathrm{Wnt}$ signaling, in which its gene expression temporally coincides with and is required for proneural gene expression. ${ }^{55}$

In wild-type flies, macrochaetes arise in stereotyped locations, and four large macrochaetes are normally observed on the scutellum region (Figure 3). However, extra macrochaetaes often appear in ark mutant flies as well as flies expressing caspase inhibitor $p 35^{56,57}$ Extra macrochetaes

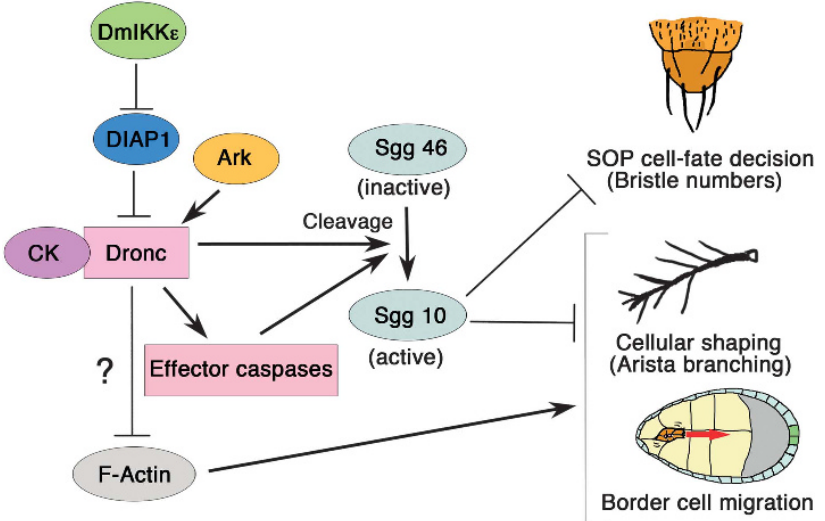

Figure 3 Non-apoptotic caspase signaling during SOP cell fate decision and cytoskeletal reorganization in Drosophila. Caspase signaling is involved in the nonapoptotic cellular processes in Drosophila, such as SOP cell fate decision and cytoskeletal reorganization during cellular shaping and border cell migration. Caspase activity is transiently and temporally controlled by the turnover of DIAP1. DIAP1 degradation is promoted by its DmIKKe-induced phosphorylation in normal development, which results in caspase activity at the level required for non-apoptotic functions. Shaggy (Sgg) protein was identified as a substrate for caspases during SOP cell specification and it becomes activated after processing by caspases (Sgg46 as inactive and Sgg10 as active form). DIAP1 positively regulates F-actin polymerization through the downregulating Dronc so that the proper amount of DIAP1 is required for border cell migration and arista morphogenesis. Dronc substrate specificity during non-apoptotic functions is conferred by the unconventional myosin CK, which physically interacts with Dronc and inactive Sgg46, and active Sgg10 negatively influences SOP cell fate determination and cytoskeletal remodeling

are also observed in dronc and $c y t-c-d$ mutant flies, ${ }^{58,59}$ raising a possibility that the apoptotic mechanism may control the macrochaete formation. Kanuka et al. ${ }^{60}$ addressed the issue of how apoptotic machinery affects macrochaete numbers. In both ark mutants and the dominant-negative form of Dronc $\left(\right.$ Dronc $\left.^{D N}\right)$-expressing flies, extra SOP cells were observed on the future scutellum area in the wing disc. Transient blocking of the caspase activity before the formation of SOP cells increased the number of macrochaetes. Intriguingly, although caspase activity was detected in proneural clusters, this was not correlated with apoptosis, suggesting that transient caspase activity negatively regulates SOP cell formation in a non-apoptotic manner. By performing genetic screening, this study further identified a kinase Shaggy (Sgg), the Drosophila GSK-3 $\beta$ ortholog as a novel substrate of active caspases. ${ }^{60}$ One of the isoforms, Sgg46, can be cleaved in a caspasedependent manner, and after the processing, it can function as an active kinase Sgg10. Sgg10 then negatively regulates $\mathrm{Wg}$ / Wnt signaling through the phosphorylation and degradation of Armadillo/ $\beta$-catenin ${ }^{60}$ or directly phosphorylating Scute and its activator Pannier, ${ }^{61}$ contributing to SOP cell specification. A subsequent study by Kuranaga et al. ${ }^{62}$ bolstered the idea that nonlethal level of caspase activity regulates precise SOP cell numbers. The Drosophila IKK-related kinase (DmIKKE) phosphorylates DIAP1 and causes its degradation, thereby regulating the caspase activity through the control of DIAP1 level. RNAi-mediated knockdown of DmIKKE, which stabilizes DIAP1 and suppresses the caspase activity, induces extra macrochaetes due to extra SOP cells. Of note, the depletion of $\mathrm{DmlKK} \varepsilon$ affects the caspase activity without contributing to naturally occurring cell death. These results suggest that 
DmlKKe determines SOP cell numbers by controlling the non-apoptotic level of transient caspase activity. ${ }^{62}$

How does transient caspase activation lead to the cleavage of a specific substrate during SOP cell fate decision? A recent study revealed that the unconventional myosin Crinkled (CK) physically interacts with Dronc, which is required for the Dronc-dependent non-apoptotic processes. ${ }^{63} \mathrm{CK}$, the Drosophila ortholog of mammalian non-muscle MYO7A, was identified from proteomic screening using the Dronc protein complex. Although CK controls different Dronc-dependent phenotypes, it does not affect naturally occurring apoptosis. Depletion of CK in the scutellum phenocopies the effect of RNAi for dronc, DmIKKE, and sgg, and the co-expression of dominant-negative form of $\mathrm{CK}\left(C K^{D N}\right)$ and DmIKKE-RNAi enhanced the extra macrochaete phenotype. These observations suggest that CK regulates the non-apoptotic function of caspases and contributes to the control of SOP cell numbers. Mechanistically, CK binds to both Dronc and inactive isoform Sgg46, but not to active Sgg10, suggesting that CK acts as an adapter that brings the substrate Sgg46 to the proximity of Dronc (Figure 3). ${ }^{63}$ Such a mechanism may confer substrate specificity of caspases, and allow the activation of downstream signaling depending on CK localization.

Caspase signaling may contribute to cell fate determination as a fine-tuning mechanism with additional factors. Indeed, the H3K9 histone methyltansferase SETDB1 has been identified to cooperatively regulate the SOP cell numbers with caspases. ${ }^{64}$ Importantly, the mechanisms that control nonapoptotic caspase signaling discovered in flies are conserved in mammals. As DmlKKe phosphorylates DIAP1, the mammalian IKK-related kinase NAK can phosphorylate XIAP. ${ }^{62}$ The mammalian CK counterpart, MYO7A, binds to caspase-8 in the presence of RIPK1 and suppresses the formation of ripoptosome complex by cleaving RIPK1. ${ }^{63}$ Given that IKKrelated kinases and RIPK1 are important for the mammalian immune system, such conserved non-apoptotic caspase signaling may perform diverse physiological functions including cell fate determination.

\section{Temporal Control of Caspase Activation in Cytoskeletal Dynamics}

Caspases are responsible for altering the morphological changes associated with apoptosis, such as cell rounding and membrane blebbing. Cytoskeletal remodeling during nonlethal events can be regulated by the caspase activity if it targets cytoskeletal regulators under strict temporal control. In mammals, during apoptosis, caspase-3 can cleave Rho-associated protein kinase 1 (ROCK1), which results in its activation and the following actin cytoskeletal rearrangement. $^{65-67}$ Caspase-3-mediated cleavage of ROCK1 is also observed during macrophage polarity formation, a non-apoptotic process. ${ }^{68}$ Moreover, caspase- 8 and caspase-11 are implicated in cell migration in non-apoptotic circumstances, ${ }^{69-71}$ suggesting that caspases are indeed the regulators for actin cytoskeleton dynamics. For controlling the caspase activity, IAPs are conserved determinants of both apoptotic and non-apoptotic functions. ${ }^{27,72}$ In particular, cellular shaping and border cell migration in Drosophila are the processes that provide mechanistic insights into the temporal control of caspase activation through IAP turnover, which influences actin cytoskeleton dynamics.

Cellular shaping and border cell migration in Drosophila. The Drosophila antenna arista has a feather-like structure that consists of a central core and a series of lateral branches (Figure 3). ${ }^{73}$ Mutant flies for DIAP1 and its antagonist Hid exhibit abnormal arista morphology, while lacking lateral branching and ectopic branching, respectively. ${ }^{74}$ Although diap1 overexpression was sufficient to induce extra branches, p35 overexpression that blocks effector caspases did not affect the arista morphology, raising the possibility that DIAP1mediated non-apoptotic caspase signaling could influence arista morphogenesis. Oshima et al. ${ }^{75}$ showed that the DIAP1-degrading kinase DmIKK $\varepsilon$ negatively regulates F-actin assembly. Expression of the dominant-negative form of DmIKKe $\left(D m / K K \varepsilon^{D N}\right)$ in arista causes an abnormal excessbranching morphology, which is suppressed by diap1 knockdown and is enhanced by diap1 overexpression, suggesting that this phenotype is sensitive to the DIAP1 dosage. Because the DIAP1 level is critical for the control of Dronc, the extra branching of arista may be attributed to the suppression of Dronc activity. Indeed, dronc mutant adult escapers exhibit extra arista branches, ${ }^{76}$ and the lateral branching phenotype caused by $D m I K K \varepsilon^{D N}$ expression is enhanced by the knockdown of dronc or its activator ark. ${ }^{75}$ A recent study further showed that the mutant allele for the unconventional myosin CK that controls Dronc activity exhibits extra arista branches in the same manner as flies expressing $D m I K K \varepsilon^{D N} .^{63}$ Although how the Dronc activity affects F-actin dynamics is still unclear, these results suggest that DIAP1-mediated control of non-apoptotic caspase signaling is crucial for the proper cellular shaping of arista (Figure 3).

Cytoskeletal dynamics regulated by non-apoptotic caspase signaling is also featured in border cell migration during Drosophila oogenesis. In the developing egg chamber, a group of cells detach from the follicle epithelium and become migratory border cells (Figure 3). ${ }^{77}$ From genetic screening, Geisbrecht and Montell ${ }^{78}$ found that the overexpression of diap1 rescues the border cell migration defect caused by a dominant-negative form of Rac $\left(\operatorname{Rac}^{D N}\right)$ expression. Mutant flies for DIAP1 also exhibit migration defects without showing cell death, suggesting that the endogenous level of DIAP1 is important for normal border cell migration. The migration defect caused by $\operatorname{Rac}^{D N}$ expression was also rescued by the overexpression of Dronc ${ }^{D N}$ or hypomorphic alleles of ark. ${ }^{78}$ In contrast, the overexpression of $D m / K K \varepsilon$ that leads to the activation of Dronc, inhibits border cell migration. ${ }^{75}$ These observations suggest that the level of DIAP1 and the subsequent control of the Dronc activity are crucial for border cell migration. During SOP cell specification, the unconventional myosin CK acts as a substrate adapter that brings the GSK-3 $\beta$ precursor Sgg46 to Dronc, facilitating its cleavage. ${ }^{63}$ The expression of $C K^{D N}$ or a non-cleavable form of Sgg46 also rescues the migration defect caused by $R a c^{D N}$ expression, suggesting that the activation of Sgg suppresses border cell migration downstream of Dronc activation. ${ }^{63}$ Although the underlying mechanism of how Sgg regulates the actual migration process remains elusive, it might affect the dynamics of the cytoskeletons or cell adhesions that are 
required for proper migration. Taken together, these results suggest that CK-regulated Dronc activity under the control of DIAP1 negatively regulates the Rac-mediated cell migration of border cells (Figure 3 ).

The above examples illuminate the role of the proper amount of DIAP1 protein or its metabolism, which is critical for the control of Dronc activity. How can DIAP1 affect both the apoptotic and non-apoptotic functions of caspases? Using a fluorescent probe for DIAP1 dynamics, which reflects its endogenous degradation, Koto et al. ${ }^{79}$ examined the protein turnover of DIAP1 in the external SOP lineage of Drosophila pupa. The SOP cells divide asymmetrically to give rise to the shaft, socket, sheath cells, and the neurons that all constitutes the shaping of this sensory organ. ${ }^{80}$ Live imagining of the SOP lineage indicated that DIAP1 turnover is controlled depending on cell type and maturity. By genetically manipulating the DIAP1 levels, this study addressed the physiological role of DIAP1 dynamics. DmIKKE-knockdown flies exhibited a delayed DIAP1 degradation in the shaft cell, which caused the shorter and thicker bristle phenotype. In contrast, the knockdown of diap1 or overexpression of rpr resulted in a defect of DIAP1 stabilization associated with the shaft cell death and subsequent bristle loss phenotype. These results suggest that temporal regulation of DIAP1 turnover determines whether caspases function in apoptosis or in cellular shaping as a non-apoptotic process. ${ }^{79}$ Given that mammalian IAPs affect cytoskeletal remodeling besides the control of caspase activity, ${ }^{72}$ similar mechanisms might play a role in switching apoptotic and non-apoptotic roles in different organisms.

\section{Caspase Activation at the Organism Level in Homeostasis and Physiology}

During animal life from embryo to adult, organisms must cope with various environmental stresses and have developed

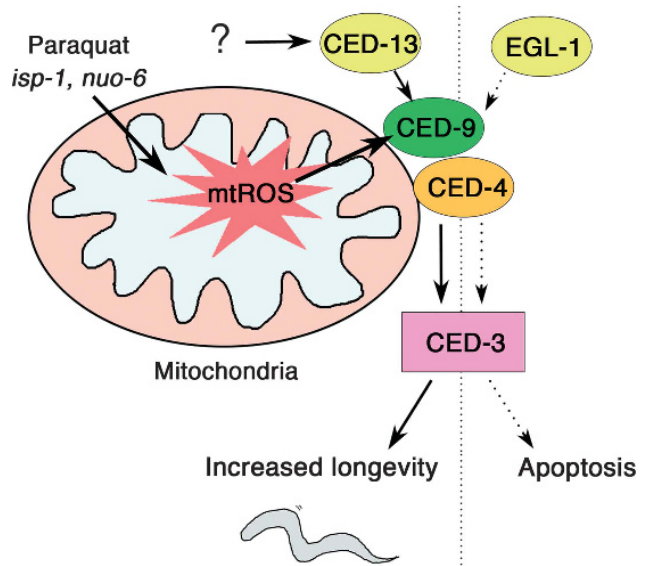

Figure 4 Apoptosis signaling affects longevity following mtROS in C. elegans. After treatment of the superoxide generator paraquat or mutations in structural components of mitochondrial respiratory chain (isp-1, nuo-6) lead to increased longevity in $C$. elegans, which is mediated by mtROS. The intrinsic apoptosis pathway (CED-9, CED-4, and CED-3) is sensitive to mtROS and is utilized as a protective mechanism for organism survival. Although the components of the core apoptosis pathway are utilized, the BH3-only protein CED-13 is required for the longevity phenotype in response to $\mathrm{mtROS}$ (left panel), instead of EGL-1, which is required for apoptosis (right panel) stress-response programs at different levels. At the cellular level, cells that have deleterious damage undergo apoptosis to remove any potentially harmful cells. In contrast, at the organism level, a coordinated stress response can enhance the resistance to further stress. ${ }^{81,82}$ Interestingly, in C. elegans, mutations in ced-3 gene confer stress resistance to a variety of stresses, such as ER and osmotic stress, ${ }^{83}$ suggesting that animal-wide stress responses are coordinated by the same signaling pathway that causes apoptosis at the cellular level. In this section, we introduce recent studies about the unexpected contribution of the apoptotic caspases to organism-level phenotypes such as ageing and whole-body development.

Increased longevity and robust development in C. elegans. As the first long-lived mutants were identified, genetic pathways that control ageing have been vigorously studied using $C$. elegans. ${ }^{84,85}$ Among the mutants that show increased longevity, missense mutations in isp-1 and nuo- 6 exhibit elevated reactive oxygen species (ROS) generation. ${ }^{86}$ The isp- 1 and nuo- 6 genes encode subunits of the complex III and complex I of the mitochondrial respiratory chain, respectively. Such a long-lived phenotype is phenocopied by treatment with a low dose of superoxide generator paraquat for wild type. These results suggest that mitochondrial ROS (mtROS) is associated with increased longevity. ${ }^{86} \mathrm{~A}$ recent study addressed the mechanism of how an increase in mtROS promotes longevity by focusing on intrinsic apoptotic signaling. ${ }^{87}$ The conserved intrinsic apoptosis pathway components, CED-9 and CED-4, are physically associated with mitochondria, and mtROS triggers the apoptotic pathway in vertebrates. ${ }^{88}$ Adding mutations in ced-9 (gain of function), ced-4 or ced-3 in isp-1 and nuo- 6 mutants significantly suppressed their prolonged life span. Similar effects on the suppression of increased longevity were observed in those mutants following exposure to paraquat. Interestingly, mutations in egl-1 that encodes the $\mathrm{BH} 3$-only protein required for apoptosis does not affect the life span of mitochondrial mutants and wild-type animal after paraquat treatment, suggesting that suppression of the increased longevity is not the results of inhibition of apoptosis. Instead, another $\mathrm{BH} 3-$ only protein CED-13 is required for the increased longevity and functions in the same pathway with CED-9, CED-4, and CED-3. These observations suggest that in the context of mitochondrial dysfunction, mtROS is sensed by the intrinsic apoptotic pathway, and this non-apoptotic function serves as a protective mechanism to maintain organism survival, leading to the increased life span (Figure 4). ${ }^{87}$

Another interesting observation came from the study of microRNAs (miRNAs) on robust animal development. miRNAmediated gene silencing is critical for diverse cellular processes by ensuring dynamic gene expression, which often works as a large complex, miRNA-induced-silencing-complex (miRISC). ${ }^{89}$ From an attempt to investigate genes that act with miRNA, Weaver et al. ${ }^{90}$ performed genome-wide RNAi screening and identified the caspase ced-3 as an interactor of ain-1, a homolog of the miRISC component. Double mutants for ced-3 and ain-1 have multiple defects in development including delays in larval growth, abnormal adult morphology, and embryonic lethality. Mutations in ain-1 do not 
affect cell death phenotypes, suggesting that the functions of ced-3 with ain-1 are non-apoptotic. Subsequent experiments revealed that ced-3 specifically functions with miRNAs in let-7family members that targets mRNA of pluripotency factors LIN-14 and LIN-28. Of note, ced-3 and ain-1 double-mutant phenotypes were suppressed by downregulating lin-14 or lin-28. These results suggest that CED-3 normally represses these pluripotency factors, leading to the hypothesis that proteolytic cleavage by CED-3 regulates their expression. In vitro assays confirmed their CED-3-dependent cleavage, and in vivo experiments demonstrated the dynamic turnover of LIN-28, which is negatively regulated by CED-3. Finally, the expression of the non-cleavable form of LIN-28 alone is sufficient to induce developmental delay and temporal cell fate patterning associated with the abundance of LIN-28, suggesting that the CED-3-dependent cleavage of LIN-28 plays a role in ensuring correct development. ${ }^{90}$ Taken together, these results suggest that the non-apoptotic caspase activity functions as a backup mechanism that allows normal development in $C$. elegans. It would be interesting to clarify whether higher organisms may have similar mechanisms to adapt to both internal abnormalities and environmental challenges, and precisely elucidate how caspase signaling integrates cellular responses to the organism level.

\section{Conclusions and Perspectives}

Recent research progress has provided a clearer view of the mechanism how killer enzymes regulate vital cellular processes. The apoptotic signaling pathway is utilized in a way that regulates the nonlethal level of caspase activity at the level of initiator caspases or by conferring substrate specificity for active caspases. In order to avoid apoptosis, it is also important to restrict the caspase activity into subcellular compartments to allow local organelle degeneration. Although it still remains elusive, caspase signaling in non-apoptotic processes must be spatiotemporally controlled, as previously shown in the cases of developmental apoptosis. ${ }^{91-94}$ Moreover, little is still known regarding what developmental signal triggers non-apoptotic caspase activation in different contexts. Dissecting the precise mechanism of when, where, and how caspase activation initiates and propagates in both apoptotic and non-apoptotic processes will provide a comprehensive view on the control of caspase activation in vivo.

Using caspase reporters in vivo, Drosophila genetic studies illustrated that the effector caspase activity has an execution threshold: cells can tolerate and survive the caspase activity below this threshold, whereas they commit suicide if the caspase activity increases beyond that threshold. ${ }^{95,96}$ Consistent with this finding, studies using the new biosensors, which detect past-caspase-3-like activities as a history, showed that large numbers of healthy cells in adult flies have experienced effector caspase activation under normal physiological conditions. ${ }^{97,98}$ This observation implies the existence of many cases of non-apoptotic caspase activation with potential novel roles. It is also possible that some cells can recover from stimulus that could cause apoptosis. This phenomenon, named anastasis, has been reported in mammalian cell cultures and in the Drosophila egg chambers under stress conditions. ${ }^{97,99}$ It is currently unclear how apoptotic cells can survive and whether anastasis is a normal developmental event with physiological significance. Looking ahead, future studies will examine the mechanism and the role of unappreciated non-apoptotic caspase activity in homeostasis and in physiology. Deciphering the physiological role of anastasis or cell recovery from apoptotic events in vivo will be another important avenue for future research. A better understanding of caspase signaling in non-apoptotic processes in vivo will lead to the precise control of the caspase activity in space and time, and the medical applications of this phenomenon may lead to potentially new treatments for diseases caused by aberrant cell death signaling.

\section{Conflict of Interest}

The authors declare no conflict of interest.

Acknowledgements. We thank members in the Kuranaga Laboratory for support and discussions. We thank Dr. M Miura for valuable inspiration and suggestions for research work on caspases and cell death. The studies of our group are supported in part by grants from the Naito Foundation (Y-IN), the Ichiro Kanehara Foundation (Y-IN), the Astellas Foundation for Research on Metabolic Disorders $(Y-I N)$, the SGH Foundation (Y-IN), the Uehara Memorial Foundation (Y-IN), the Takeda Science Foundation (EK), the Japan Foundation for Applied Enzymology (EK), MEXT KAKENHI Grant Number JP26114003 (EK), and the JSPS KAKENHI Grant Numbers JP16H06634 (Y-IN), JP24687027 (EK), and JP16H04800 (EK).

1. Taylor RC, Cullen SP, Martin SJ. Apoptosis: controlled demolition at the cellular level. Nat Rev Mol Cell Biol 2008; 9: 231-241.

2. Jacobson MD, Weil M, Raff MC. Programmed cell death in animal development. Cell 1997; 88: 347-354

3. Vaux DL, Korsmeyer SJ. Cell death in development. Cell 1999; 96: 245-254.

4. Fuchs Y, Steller H. Programmed cell death in animal development and disease. Cell 2011; 147: $742-758$.

5. Suzanne M, Steller H. Shaping organisms with apoptosis. Cell Death Differ 2013; 20: 669-675.

6. Perez-Garijo A, Steller H. Spreading the word: non-autonomous effects of apoptosis during development, regeneration and disease. Development 2015; 142: 3253-3262.

7. Kawamoto Y, Nakajima YI, Kuranaga E. Apoptosis in cellular society: communication between apoptotic cells and their neighbors. Int J Mol Sci 2016; 17: E2144.

8. Ranger AM, Malynn BA, Korsmeyer SJ. Mouse models of cell death. Nat Genet 2001; 28: 113-118.

9. Evan GI, Vousden KH. Proliferation, cell cycle and apoptosis in cancer. Nature 2001; 411: 342-348.

10. Rock KL, Kono H. The inflammatory response to cell death. Annu Rev Pathol 2008; 3 : 99-126.

11. Cotter TG. Apoptosis and cancer: the genesis of a research field. Nat Rev Cancer 2009; 9 : 501-507.

12. Abraham MC, Shaham S. Death without caspases, caspases without death. Trends Cell Biol 2004; 14: 184-193.

13. Feinstein-Rotkopf $Y$, Arama E. Can't live without them, can live with them: roles of caspases during vital cellular processes. Apoptosis 2009; 14: 980-995.

14. Galluzzi L, Joza N, Tasdemir E, Maiuri MC, Hengartner M, Abrams JM et al. No death without life: vital functions of apoptotic effectors. Cell Death Differ 2008; 15: 1113-1123.

15. Galluzzi L, Kepp O, Trojel-Hansen C, Kroemer G. Non-apoptotic functions of apoptosisregulatory proteins. EMBO Rep 2012; 13: 322-330.

16. Kuranaga $\mathrm{E}$. Beyond apoptosis: caspase regulatory mechanisms and functions in vivo. Genes Cells 2012; 17: 83-97.

17. Kuranaga E, Miura M. Nonapoptotic functions of caspases: caspases as regulatory molecules for immunity and cell-fate determination. Trends Cell Biol 2007; 17: 135-144.

18. Lamkanfi M, Festjens N, Declercq W, Vanden Berghe T, Vandenabeele P. Caspases in cell survival, proliferation and differentiation. Cell Death Differ 2007; 14: 44-55.

19. Yi CH, Yuan J. The Jekyll and Hyde functions of caspases. Dev Cell 2009; 16: 21-34.

20. Ryoo HD, Bergmann A. The role of apoptosis-induced proliferation for regeneration and cancer. Cold Spring Harb Perspect Biol 2012; 4: a008797.

21. Bilder D. The maturation of development. Dev Cell 2016; 38: 569-570.

22. Ellis HM, Horvitz HR. Genetic control of programmed cell death in the nematode C. elegans. Cell 1986; 44: 817-829.

23. Conradt B, Wu YC, Xue D. Programmed cell death during caenorhabditis elegans development. Genetics 2016; 203: 1533-1562. 
24. Mcllwain DR, Berger T, Mak TW. Caspase functions in cell death and disease. Cold Spring Harb Perspect Biol 2013; 5: a008656.

25. Crawford ED, Wells JA. Caspase substrates and cellular remodeling. Annu Rev Biochem 2011; 80: 1055-1087.

26. Hay BA, Huh JR, Guo M. The genetics of cell death: approaches, insights and opportunities in Drosophila. Nat Rev Genet 2004; 5: 911-922.

27. Vasudevan D, Ryoo HD. Regulation of cell death by IAPs and their antagonists. Curr Top Dev Biol 2015; 114: 185-208.

28. Sandu C, Ryoo HD, Steller H. Drosophila IAP antagonists form multimeric complexes to promote cell death. J Cell Biol 2010; 190: 1039-1052.

29. Schile AJ, Garcia-Fernandez M, Steller H. Regulation of apoptosis by XIAP ubiquitin-ligase activity. Genes Dev 2008; 22: 2256-2266.

30. Bader M, Steller H. Regulation of cell death by the ubiquitin-proteasome system. Curr Opin Cell Biol 2009; 21: 878-884

31. Arya R, White K. Cell death in development: signaling pathways and core mechanisms. Semin Cell Dev Biol 2015; 39: 12-19.

32. Ishizaki Y, Jacobson MD, Raff MC. A role for caspases in lens fiber differentiation. J Cell Biol 1998; 140: 153-158.

33. Weber GF, Menko AS. The canonical intrinsic mitochondrial death pathway has a nonapoptotic role in signaling lens cell differentiation. J Biol Chem 2005; 280: 22135-22145.

34. Fabian L, Brill JA. Drosophila spermiogenesis: big things come from little packages. Spermatogenesis 2012; 2: 197-212.

35. Noguchi T, Miller KG. A role for actin dynamics in individualization during spermatogenesis in Drosophila melanogaster. Development 2003; 130: 1805-1816.

36. Arama $\mathrm{E}$, Agapite J, Steller H. Caspase activity and a specific cytochrome $\mathrm{C}$ are required for sperm differentiation in Drosophila. Dev Cell 2003; 4: 687-697.

37. Arama E, Bader M, Srivastava M, Bergmann A, Steller H. The two Drosophila cytochrome C proteins can function in both respiration and caspase activation. EMBO J 2006; 25: 232-243.

38. Huh JR, Vernooy SY, Yu H, Yan N, Shi Y, Guo M et al. Multiple apoptotic caspase cascades are required in nonapoptotic roles for Drosophila spermatid individualization. PLoS Biol 2004; 2: E15.

39. Muro I, Berry DL, Huh JR, Chen CH, Huang H, Yoo SJ et al. The Drosophila caspase Ice is important for many apoptotic cell deaths and for spermatid individualization, a nonapoptotic process. Development 2006; 133: 3305-3315.

40. Chew SK, Chen P, Link N, Galindo KA, Pogue K, Abrams JM. Genome-wide silencing in Drosophila captures conserved apoptotic effectors. Nature 2009; 460: 123-127.

41. D'Brot A, Chen P, Vaishnav M, Yuan S, Akey CW, Abrams JM. Tango7 directs cellular remodeling by the Drosophila apoptosome. Genes Dev 2013; 27: 1650-1655.

42. Arama E, Bader M, Rieckhof GE, Steller H. A ubiquitin ligase complex regulates caspase activation during sperm differentiation in Drosophila. PLOS Biol 2007; 5: e251.

43. Kaplan Y, Gibbs-Bar L, Kalifa Y, Feinstein-Rotkopf Y, Arama E. Gradients of a ubiquitin E3 ligase inhibitor and a caspase inhibitor determine differentiation or death in spermatids. Dev Cell 2010; 19: 160-173.

44. Aram L, Braun T, Braverman C, Kaplan Y, Ravid L, Levin-Zaidman S et al. A Krebs cycle component limits caspase activation rate through mitochondrial surface restriction of $C R L$ activation. Dev Cell 2016; 37: 15-33.

45. Kissel H, Georgescu MM, Larisch S, Manova K, Hunnicutt GR, Steller H. The Sept4 septin locus is required for sperm terminal differentiation in mice. Dev Cell 2005; 8: $353-364$

46. Blanco-Rodriguez J, Martinez-Garcia C. Apoptosis is physiologically restricted to a specialized cytoplasmic compartment in rat spermatids. Biol Reprod 1999; 61: 1541-1547.

47. Wang S, Zheng H, Esaki Y, Kelly F, Yan W. Cullin3 is a KLHL10-interacting protein preferentially expressed during late spermiogenesis. Biol Reprod 2006; 74: 102-108.

48. Miura M, Chen XD, Allen MR, Bi Y, Gronthos S, Seo BM et al. A crucial role of caspase-3 in osteogenic differentiation of bone marrow stromal stem cells. J Clin Invest 2004; 114: 1704-1713.

49. Fernando $P$, Brunette $S$, Megeney LA. Neural stem cell differentiation is dependent upon endogenous caspase 3 activity. FASEB J 2005; 19: 1671-1673.

50. Fujita J, Crane AM, Souza MK, Dejosez M, Kyba M, Flavell RA et al. Caspase activity mediates the differentiation of embryonic stem cells. Cell Stem Cell 2008; 2: 595-601.

51. Janzen V, Fleming HE, Riedt T, Karlsson G, Riese MJ, Lo Celso C et al. Hematopoietic stem cell responsiveness to exogenous signals is limited by caspase-3. Cell Stem Cell 2008; 2 : 584-594.

52. Li F, He Z, Shen J, Huang Q, Li W, Liu X et al. Apoptotic caspases regulate induction of iPSCs from human fibroblasts. Cell Stem Cell 2010; 7: 508-520.

53. Calleja M, Renaud O, Usui K, Pistillo D, Morata G, Simpson P. How to pattern an epithelium: lessons from achaete-scute regulation on the notum of Drosophila. Gene 2002; 292: 1-12.

54. Troost T, Schneider M, Klein T. A re-examination of the selection of the sensory organ precursor of the bristle sensilla of Drosophila melanogaster. PLoS Genet 2015; 11: e1004911.

55. Phillips RG, Whittle JR. wingless expression mediates determination of peripheral nervous system elements in late stages of Drosophila wing disc development. Development 1993; 118: $427-438$.

56. Kanuka H, Sawamoto K, Inohara N, Matsuno K, Okano H, Miura M. Control of the cell death pathway by Dapaf-1, a Drosophila Apaf-1/CED-4-related caspase activator. Mol Cell 1999; 4: 757-769.
57. Rodriguez A, Oliver H, Zou H, Chen P, Wang X, Abrams JM. Dark is a Drosophila homologue of Apaf-1/CED-4 and functions in an evolutionarily conserved death pathway. Nat Cell Biol 1999; 1: 272-279.

58. Chew SK, Akdemir F, Chen P, Lu WJ, Mills K, Daish T et al. The apical caspase dronc governs programmed and unprogrammed cell death in Drosophila. Dev Cell 2004; 7 : 897-907.

59. Mendes CS, Arama E, Brown S, Scherr H, Srivastava M, Bergmann A et al. Cytochrome c-d regulates developmental apoptosis in the Drosophila retina. EMBO Rep 2006; 7: 933-939.

60. Kanuka H, Kuranaga E, Takemoto K, Hiratou T, Okano H, Miura M. Drosophila caspase transduces Shaggy/GSK-3beta kinase activity in neural precursor development. EMBO J 2005; 24: 3793-3806.

61. Yang M, Hatton-Ellis E, Simpson P. The kinase Sgg modulates temporal development of macrochaetes in Drosophila by phosphorylation of Scute and Pannier. Development 2012; 139: 325-334

62. Kuranaga $\mathrm{E}$, Kanuka $\mathrm{H}$, Tonoki $\mathrm{A}$, Takemoto $\mathrm{K}$, Tomioka $\mathrm{T}$, Kobayashi $\mathrm{M}$ et al. Drosophila IKK-related kinase regulates nonapoptotic function of caspases via degradation of IAPs. Cell 2006; 126: 583-596.

63. Orme MH, Liccardi G, Moderau N, Feltham R, Wicky-John S, Tenev T et al. The unconventional myosin CRINKLED and its mammalian orthologue MYO7A regulate caspases in their signalling roles. Nat Commun 2016; 7: 10972.

64. Shinoda N, Obata F, Zhang L, Miura M. Drosophila SETDB1 and caspase cooperatively finetune cell fate determination of sensory organ precursor. Genes Cells 2016; 21: 378-386.

65. Coleman ML, Sahai EA, Yeo M, Bosch M, Dewar A, Olson MF. Membrane blebbing during apoptosis results from caspase-mediated activation of ROCK I. Nat Cell Biol 2001; 3 . $339-345$.

66. Sebbagh M, Renvoize C, Hamelin J, Riche N, Bertoglio J, Breard J. Caspase-3-mediated cleavage of ROCK I induces MLC phosphorylation and apoptotic membrane blebbing Nat Cell Biol 2001; 3: 346-352.

67. Chang J, Xie M, Shah VR, Schneider MD, Entman ML, Wei $L$ et al. Activation of Rho-associated coiled-coil protein kinase 1 (ROCK-1) by caspase-3 cleavage plays an essential role in cardiac myocyte apoptosis. Proc Natl Acad Sci USA 2006; 103 14495-14500.

68. Liu Y, Minze LJ, Mumma L, Li XC, Ghobrial RM, Kloc M. Mouse macrophage polarity and ROCK1 activity depend on RhoA and non-apoptotic Caspase 3. Exp Cell Res 2016; 341 225-236.

69. Helfer B, Boswell BC, Finlay D, Cipres A, Vuori K, Bong Kang T et al. Caspase-8 promotes cell motility and calpain activity under nonapoptotic conditions. Cancer Res 2006; 66 : 4273-4278.

70. Li J, Brieher WM, Scimone ML, Kang SJ, Zhu H, Yin $\mathrm{H}$ et al. Caspase-11 regulates cell migration by promoting Aip1-Cofilin-mediated actin depolymerization. Nat Cell Biol 2007; 9 : $276-286$.

71. Torres VA, Mielgo A, Barbero S, Hsiao R, Wilkins JA, Stupack DG. Rab5 mediates caspase8-promoted cell motility and metastasis. Mol Biol Cell 2010; 21: 369-376.

72. Fulda S. Regulation of cell migration, invasion and metastasis by IAP proteins and their antagonists. Oncogene 2014; 33: 671-676.

73. He B, Adler PN. Cellular mechanisms in the development of the Drosophila arista. Mech Dev 2001; 104: 69-78.

74. Cullen K, McCall K. Role of programmed cell death in patterning the Drosophila antennal arista. Dev Biol 2004; 275: 82-92.

75. Oshima K, Takeda M, Kuranaga E, Ueda R, Aigaki T, Miura M et al. IKK epsilon regulates $F$ actin assembly and interacts with Drosophila IAP1 in cellular morphogenesis. Curr Biol 2006 16: 1531-1537.

76. Baum JS, Arama E, Steller H, McCall K. The Drosophila caspases Strica and Dronc function redundantly in programmed cell death during oogenesis. Cell Death Differ 2007; 14 1508-1517.

77. Montell DJ. Border-cell migration: the race is on. Nat Rev Mol Cell Biol 2003; 4: 13-24.

78. Geisbrecht ER, Montell DJ. A role for Drosophila IAP1-mediated caspase inhibition in Racdependent cell migration. Cell 2004; 118: 111-125.

79. Koto A, Kuranaga E, Miura M. Temporal regulation of Drosophila IAP1 determines caspase functions in sensory organ development. J Cell Biol 2009; 187: 219-231.

80. Schweisguth F. Asymmetric cell division in the Drosophila bristle lineage: from the polarization of sensory organ precursor cells to Notch-mediated binary fate decision. Wiley Interdiscip Rev Dev Biol 2015; 4: 299-309.

81. Cypser JR, Tedesco P, Johnson TE. Hormesis and aging in Caenorhabditis elegans. Exp Gerontol 2006; 41: 935-939.

82. Pickering AM, Staab TA, Tower J, Sieburth D, Davies KJ. A conserved role for the $20 \mathrm{~S}$ proteasome and Nrf2 transcription factor in oxidative stress adaptation in mammals Caenorhabditis elegans and Drosophila melanogaster. J Exp Biol 2013; 216(Pt 4): 543-553.

83. Judy ME, Nakamura A, Huang A, Grant $\mathrm{H}$, McCurdy $\mathrm{H}$, Weiberth $\mathrm{KF}$ et al. A shift to organismal stress resistance in programmed cell death mutants. PLOS Genet 2013; 9 : e1003714.

84. Kenyon $C$. The first long-lived mutants: discovery of the insulin/GF-1 pathway for ageing Philos Trans R Soc Lond B Biol Sci 2011; 366: 9-16.

85. Shore $D E$, Ruvkun $G$. A cytoprotective perspective on longevity regulation. Trends Cell Biol 2013; 23: 409-420.

86. Yang W, Hekimi S. A mitochondrial superoxide signal triggers increased longevity in Caenorhabditis elegans. PLOS Biol 2010; 8: e1000556. 
87. Yee C, Yang W, Hekimi S. The intrinsic apoptosis pathway mediates the pro-longevity response to mitochondrial ROS in C. elegans. Cell 2014; 157: 897-909.

88. Wang C, Youle RJ. The role of mitochondria in apoptosis. Annu Rev Genet 2009; 43: 95-118.

89. Fabian MR, Sonenberg N. The mechanics of miRNA-mediated gene silencing: a look under the hood of miRISC. Nat Struct Mol Biol 2012; 19: 586-593.

90. Weaver BP, Zabinsky R, Weaver YM, Lee ES, Xue D, Han M. CED-3 caspase acts with miRNAs to regulate non-apoptotic gene expression dynamics for robust development in C. elegans. Elife 2014; 3: e04265.

91. Takemoto K, Kuranaga E, Tonoki A, Nagai T, Miyawaki A, Miura M. Local initiation of caspase activation in Drosophila salivary gland programmed cell death in vivo. Proc Natl Acad Sci USA 2007; 104: 13367-13372.

92. Kuranaga E, Matsunuma T, Kanuka H, Takemoto K, Koto A, Kimura K et al. Apoptosis controls the speed of looping morphogenesis in Drosophila male terminalia. Development 2011; 138: 1493-1499.

93. Nakajima Y, Kuranaga E, Sugimura K, Miyawaki A, Miura M. Nonautonomous apoptosis is triggered by local cell cycle progression during epithelial replacement in Drosophila. Mol Cell Biol 2011; 31: 2499-2512.

94. Yamaguchi Y, Shinotsuka N, Nonomura K, Takemoto K, Kuida K, Yosida H et al. Live imaging of apoptosis in a novel transgenic mouse highlights its role in neural tube closure. J Cell Biol 2011; 195: 1047-1060.

95. Florentin A, Arama E. Caspase levels and execution efficiencies determine the apoptotic potential of the cell. J Cell Biol 2012; 196: 513-527.

96. Ribeiro PS, Kuranaga E, Tenev T, Leulier F, Miura M, Meier P. DIAP2 functions as a mechanism-based regulator of drICE that contributes to the caspase activity threshold in living cells. J Cell Biol 2007; 179: 1467-1480.
97. Tang HL, Tang HM, Fung MC, Hardwick JM. In vivo CaspaseTracker biosensor system for detecting anastasis and non-apoptotic caspase activity. Sci Rep 2015; 5: 9015.

98. Ding AX, Sun G, Argaw YG, Wong JO, Easwaran S, Montell DJ. CasExpress reveals widespread and diverse patterns of cell survival of caspase-3 activation during development in vivo. Elife 2016; 5: e10936.

99. Tang HL, Tang HM, Mak KH, Hu S, Wang SS, Wong KM et al. Cell survival, DNA damage, and oncogenic transformation after a transient and reversible apoptotic response. Mol Biol Cell 2012; 23: 2240-2252.

(c) (1) () $\odot$ This work is licensed under a Creative Commons Attribution-NonCommercial-NoDerivs 4.0 International License. The images or other third party material in this article are included in the article's Creative Commons license, unless indicated otherwise in the credit line; if the material is not included under the Creative Commons license, users will need to obtain permission from the license holder to reproduce the material. To view a copy of this license, visit http://creativecommons.org/licenses/by-nc-nd/4.0/

(C) The Author(s) 2017 